\title{
DECOMPOSIÇÃO ÁCIDA ASSISTIDA POR ULTRASSOM PARA A DETERMINAÇÃO DE Cu, Fe, Mg E Zn POR F AAS EM CERÂMICAS DE USO DOMÉSTICO
}

\author{
Eliézer Quadro Oreste", Alexander Ossanes de Souza\#, Camila Corrêa Pereira\#, Mariana Antunes Vieira ${ }^{\#}$ e Anderson \\ Schwingel Ribeiro*,\# \\ Centro de Ciências Químicas, Farmacêuticas e de Alimentos, Universidade Federal de Pelotas, Campus Capão do Leão, 96160- \\ 000 Capão do Leão - RS, Brasil
}

Recebido em 27/09/2016; aceito em 28/11/2016; publicado na web em 10/02/2017

\begin{abstract}
ACID DECOMPOSITION ASSISTED BY ULTRASOUND FOR DETERMINATION OF Cu, Fe, Mg AND Zn BY F AAS IN DOMESTIC CERAMICS. This paper describes a sample preparation method for domestic ceramics, based on the acid decomposition assisted by ultrasound for further determination of $\mathrm{Cu}, \mathrm{Fe}, \mathrm{Mg}$ and $\mathrm{Zn}$ by Flame Atomic Absorption Spectrometry technique. The main optimized conditions were: particle size $(25 \mu \mathrm{m})$; sample mass $(25 \mathrm{mg})$ and the acid volume $\left(0.5 \mathrm{~mL}\right.$ of $\mathrm{HNO}_{3}$ and $0.5 \mathrm{~mL}$ of $\mathrm{HF}$ ). The samples were placed in an ultrasonic bath for 2 hours at $40{ }^{\circ} \mathrm{C}$. The method was employed for eight commercial samples of domestic ceramic, which showed different concentrations for all analytes investigated, showing the existing variety of raw materials. The method showed good accuracy and precision, attested by the addition and recovery test, with values of 87 to $112 \%$ for all analytes and relative standard deviation lower than $3.0 \%$.
\end{abstract}

Keywords: domestic ceramics; metals; acid decomposition; ultrasound; F AAS.

\section{INTRODUÇÃO}

As cerâmicas são definidas como sólidos inorgânicos com estrutura cristalina e que são formadas através de ligações químicas entre metais e não metais..$^{1,2}$ Embora algumas cerâmicas sejam quebradiças, elas possuem, dentre outras características, elevada dureza e resistência a altas temperaturas, o que lhes conferem inúmeras aplicações em vários setores, tais como: elétrico, magnético, ótico, automotivo, biomédico, construção, químico e doméstico. ${ }^{3}$ De modo geral, os processos de fabricação das cerâmicas variam de acordo com o tipo de peça ou de material desejado, os quais envolvem a preparação da matéria prima, o tratamento térmico e o acabamento final. ${ }^{4}$

A primeira aplicação dos materiais cerâmicos ocorreu no período neolítico, em que eram fabricados de argila e cuja utilização servia para a conservação de água e de alimentos. ${ }^{5,6}$ Dentre as suas utilidades, destacam-se no setor doméstico, em que, atualmente, são utilizados principalmente em louças de mesa (aparelhos de jantar, jogos de xícaras, utensílios para acondicionar alimentos e outros). ${ }^{7}$

São fabricados em quase todos os países, a partir de matéria prima local. No Brasil, existem inúmeras indústrias responsáveis pela produção de materiais cerâmicos para uso doméstico, as quais estão predominantemente distribuídas nas regiões Sul e Sudeste. ${ }^{7,8}$ Para o acabamento, alguns materiais cerâmicos passam por uma etapa de recobrimento com esmalte, que propicia um aumento da resistência química e mecânica, além de diminuir a permeabilidade. $\mathrm{O}$ acabamento, ademais, melhora o aspecto estético, já que é possível obter materiais com diferentes desenhos e cores.

A cerâmica final pode apresentar uma gama de elementos metálicos em sua composição, os quais não são constantemente monitorados durante o seu processo de produção. A falta de uniformidade com relação à composição química dos materiais cerâmicos é indesejada para a sua aplicabilidade no setor doméstico, já que pode haver, por exemplo, possibilidade de migração dos elementos para os alimentos e bebidas. ${ }^{2,9,10}$

*e-mail: andersonsr@pq.cnpq.br

\#Programa de Pós-Graduação em Química
No Brasil, ainda não existem normas para o controle das concentrações de metais nas cerâmicas de uso doméstico. Esse fato resultou na chamada MCTI/CNPq/ANVISA N ${ }^{\circ}$ 23/2012, em que um dos objetivos foi receber propostas que viabilizassem avaliar a concentração de metais em utensílios usados para o preparo de alimentos (porcelanas), incluindo produtos importados. Diante disso, fica evidente a necessidade e a importância do desenvolvimento de metodologias analíticas para a determinação de metais em utensílios cerâmicos de uso doméstico.

Para a determinação de metais, a técnica de espectrometria de absorção atômica em chama (FAAS) caracteriza-se por apresentar uma boa sensibilidade, seletividade e baixo custo. Essa técnica geralmente utiliza um nebulizador pneumático para introdução de amostras, o que requer que estas estejam em solução e livre de materiais particulados que possam causar entupimento do sistema. ${ }^{11,12}$

Para definir o método de preparo mais adequado, e que promova uma completa decomposição, é necessário conhecer previamente a composição química da amostra. ${ }^{13-15}$ Os materiais cerâmicos geralmente são fabricados a partir de matérias-primas à base de silicatos, o que se torna vantajoso devido à grande abundância e ao baixo custo desses materiais. ${ }^{16}$ Para a decomposição de amostras ricas em silício, geralmente é necessário uma combinação de ácidos envolvendo o ácido fluorídrico (HF). ${ }^{17-22}$ Contudo, a quantidade residual de HF nas soluções das amostras a serem analisadas deve ser mascarada com o ácido bórico $\left(\mathrm{H}_{3} \mathrm{BO}_{3}\right)$ para evitar problemas como a corrosão de algumas peças instrumentais que contenham silício em sua composição. ${ }^{18,23}$

Como fonte de energia para os procedimentos de preparo de amostra, uma alternativa amplamente utilizada é o uso de ondas ultrassônicas que podem conduzir processos de extração, dissolução e decomposição quando aplicadas a amostras sólidas em pó, que se encontram dispersas em um meio líquido apropriado. ${ }^{24-26}$ Conforme descrito por Krug e colaboradores, ${ }^{14}$ "os principais efeitos do uso das ondas ultrassônicas em processos de decomposição são a desagregação e ruptura do material pela ação de microjatos aumentando a superfície de reação, o aumento da atividade química a partir de espécies presentes no solvente, a renovação do solvente na interface 
solvente-soluto sólido e, até mesmo, a fusão do sólido devido às temperaturas extremas atingidas no momento do colapso das bolhas de cavitação".

Considerando a importância da determinação de elementos metálicos em utensílios cerâmicos para uso doméstico, o presente trabalho tem por objetivo, portanto, apresentar um método envolvendo a decomposição ácida assistida por ultrassom para posterior determinação de $\mathrm{Cu}, \mathrm{Fe}, \mathrm{Mg}$ e $\mathrm{Zn}$ nessas amostras pela técnica de espectrometria de absorção atômica em chama (F AAS).

\section{PARTE EXPERIMENTAL}

\section{Instrumentação}

Para a determinação dos elementos $\mathrm{Cu}, \mathrm{Fe}, \mathrm{Mg}$ e $\mathrm{Zn}$, foi utilizado um espectrômetro de absorção atômica com chama da Perkin Elmer, modelo AAnalyst 200 (Shelton, CT, EUA), equipado com lâmpadas de cátodo oco para cada analito (Perkin Elmer, Shelton, CT, EUA) e uma lâmpada de arco de deutério para correção do sinal de fundo. A chama foi composta por uma mistura de ar (gás oxidante) e acetileno (gás combustível) (Linde, Barueri, SP, Brasil). As condições operacionais estão apresentadas na Tabela 1 .

Tabela 1. Condições operacionais para determinação de $\mathrm{Cu}, \mathrm{Fe}, \mathrm{Mg}$ e $\mathrm{Zn}$ por F AAS

\begin{tabular}{lcccc}
\hline Parâmetros & $\mathrm{Cu}$ & $\mathrm{Fe}$ & $\mathrm{Mg}$ & $\mathrm{Zn}$ \\
\hline Comprimento de onda $(\mathrm{nm})$ & 324,75 & 248,33 & 285,21 & 213,86 \\
Fenda espectral $(\mathrm{nm})$ & 2,7 & 1,8 & 2,7 & 2,7 \\
Corrente da lâmpada $(\mathrm{mA})$ & 15 & 30 & 6 & 15 \\
Vazão de ar $\left(\mathrm{L} \mathrm{min}^{-1}\right)$ & 10 & 10 & 10 & 10 \\
Vazão de acetileno $\left(\mathrm{L} \mathrm{min}^{-1}\right)$ & 2,5 & 2,5 & 2,5 & 2,5 \\
\hline
\end{tabular}

As amostras foram cominuídas em um moinho de bolas, modelo MM 400 (Retsch, Alemanha), com copos de moagem fabricados com carbeto de tungstênio e, posteriormente, peneiradas em diferentes granulometrias em um agitador de peneiras (A Bronzinox, Brasil). Além disso, as amostras foram pesadas em uma balança analítica Ohaus Adventurer, modelo Ar 2140 (Pine Brook, NJ, EUA), com uma resolução de $0,1 \mathrm{mg}$ e tara de $210 \mathrm{~g}$. No preparo das amostras por decomposição ácida foi utilizado um banho ultrassônico, modelo Elmasonic S 40 (H) (Elma, Alemanha), com potência de $560 \mathrm{~W}$ e frequência de $37 \mathrm{kHz}$, além de um bloco digestor, modelo MA 850 (Marconi, Brasil), para uso com tubos e bombas de politetrafluoretileno (PTFE) com até $50 \mathrm{~mm}$ de diâmetro.

\section{Materiais e reagentes}

Todos os reagentes utilizados foram de grau analítico e as soluções empregadas foram preparadas com água desionizada, obtida a partir de um sistema de destilação de vidro (MA078/5, Marconi, SP, Brasil) e, posteriormente, eluída em um desionizador (CS1800 Evolution, Permution, PR, Brasil). As soluções de calibração para os analitos foram preparadas em meio ácido, idêntico ao utilizado no preparo das amostras, a partir da diluição de uma solução estoque contendo $1000 \mathrm{mg} \mathrm{L}^{-1}$ (Merck, Darmstadt, Alemanha) em água desionizada.

Para o preparo das amostras, foi utilizado ácido nítrico, $\mathrm{HNO}_{3}$ $65 \% \mathrm{~m} / \mathrm{m}$ (Vetec, RJ, Brasil), bidestilado abaixo do seu ponto de ebulição em um destilador de quartzo MA-075 (Marconi, SP, Brasil). Nessa etapa, também foi utilizado ácido fluorídrico $40 \% \mathrm{~m} / \mathrm{m}, \mathrm{HF}$ (Vetec, RJ, Brasil) e ácido bórico, $\mathrm{H}_{3} \mathrm{BO}_{3} 99,5 \% \mathrm{~m} / \mathrm{m}$ (SigmaAldrich, EUA). Para a determinação de $\mathrm{Mg}$ por F AAS foi utilizada uma solução de Schinkel composta por $\mathrm{CsCl} 1 \% \mathrm{~m} / \mathrm{v}$ e $\mathrm{LaCl}_{3} 10 \%$ $\mathrm{m} / \mathrm{v}$ (Fluka, Alemanha) como supressor de ionização e formação de óxidos, respectivamente.

Os materiais utilizados para o preparo das soluções, padrões e decomposição das amostras foram lavados com água e detergente e, após secarem a temperatura ambiente, foram imersos em uma solução de $\mathrm{HNO}_{3} 10 \%$ v/v por $48 \mathrm{~h}$. Na etapa seguinte, foram enxaguados com água desionizada e secos à temperatura ambiente antes de serem utilizados.

\section{Amostras e métodos de preparo}

Para o desenvolvimento do método de decomposição ácida assistida por ultrassom e verificação das concentrações dos analitos, foram utilizadas oito amostras de louças cerâmicas importadas e de modelos diferentes, comercializadas na cidade de Pelotas/RS. Essas amostras foram denominadas como: caneca amarela (A); caneca branca com desenhos coloridos (B); xícara branca com desenhos coloridos (C); caneca azul com desenhos coloridos (D); caneca com listras em verde e marrom $(\mathrm{E})$; xícara branca $(\mathrm{F})$; caneca preta $(\mathrm{G})$ e caneca verde $(\mathrm{H})$.

Em um primeiro momento, todas as amostras passaram por um tratamento preliminar, em que foram lavadas e secas para, posteriormente, serem fragmentadas e transformadas em pó utilizando-se, para tanto, o moinho de bolas. Em seguida, foram peneiradas em um sistema de agitação para obtenção de partículas com granulometrias que variaram de $>150 \mathrm{a}<25 \mu \mathrm{m}$.

Com a finalidade de avaliar a exatidão dos resultados obtidos pelo método de decomposição ácida assistida por ultrassom, as amostras também foram preparadas por outros dois métodos de decomposição em meio ácido: um em sistema aberto (SA) e outro em sistema fechado (SF). Todos os métodos empregados são descritos a seguir.

\section{Procedimento 1: Decomposição ácida assistida por ultrassom (US)}

Para a decomposição, foram pesados aproximadamente $25 \mathrm{mg}$ de amostra, com granulometria inferior a $25 \mu \mathrm{m}$, diretamente em frascos volumétricos de polipropileno (PP) e, posteriormente, fez-se a adição de uma mistura ácida contendo $0,50 \mathrm{~mL}$ de $\mathrm{HNO}_{3}$ e 0,50 $\mathrm{mL}$ de HF. Subsequentemente, as soluções foram colocadas em um banho ultrassônico a uma temperatura de $40{ }^{\circ} \mathrm{C}$ e sonificadas por 2 horas. Após esse período, as soluções resultantes mostraram-se totalmente límpidas. O volume final de 20,0 mL foi completado com uma solução de $\mathrm{H}_{3} \mathrm{BO}_{3} 4,5 \% \mathrm{~m} / \mathrm{v}$ a fim de complexar o $\mathrm{HF}$ remanescente.

A quantidade necessária de $\mathrm{HF}$ foi calculada seguindo a estequiometria da reação, na qual para cada $1 \mathrm{~mL}$ de $\mathrm{HF} 40 \% \mathrm{~m} / \mathrm{m}$, seriam necessários $8 \mathrm{~mL}$ da solução $4,5 \% \mathrm{~m} / \mathrm{v}$ de $\mathrm{H}_{3} \mathrm{BO}_{3}$ para a reação entre o $\mathrm{HF}$ e o $\mathrm{H}_{3} \mathrm{BO}_{3}$. Entretanto, em todos os métodos de preparo utilizados neste trabalho foi adicionado um excesso de $\mathrm{H}_{3} \mathrm{BO}_{3}$, visto que não apresenta prejuízo aos resultados das determinações, apenas garante que todo HF esteja complexado evitando, desse modo, danificar o sistema de introdução de amostra do espectrômetro.

\section{Procedimento 2: Decomposição ácida em sistema aberto (SA)}

Este foi adaptado a partir do procedimento descrito por Santos e colaboradores. ${ }^{22}$ Pesaram-se, aproximadamente, $100 \mathrm{mg}$ de amostra, com granulometria inferior a $25 \mu \mathrm{m}$, diretamente em frascos de PTFE e, posteriormente, fez-se a adição de uma mistura ácida contendo 3,0 $\mathrm{mL}$ de $\mathrm{HNO}_{3}$ e $1,0 \mathrm{~mL}$ de HF. Os frascos abertos foram encaminhados diretamente a um bloco digestor aquecido a uma temperatura de $150{ }^{\circ} \mathrm{C}$ no qual permaneceram até quase a secura dos ácidos. Após 
arrefecimento até a temperatura ambiente, a solução foi transferida para frascos volumétricos de $\mathrm{PP}$ e o volume de $30,0 \mathrm{~mL}$ foi completado com uma solução de $\mathrm{H}_{3} \mathrm{BO}_{3} 4,5 \% \mathrm{~m} / \mathrm{v}$.

\section{Procedimento 3: Decomposição ácida em sistema fechado (SF)}

Para a decomposição ácida em sistema fechado, foi seguido o procedimento descrito pela Environmental Protection Agency (EPA), ${ }^{27}$ com a substituição do forno micro-ondas pelo bloco digestor. Foram pesados aproximadamente $250 \mathrm{mg}$ de amostra, com granulometria inferior a $25 \mu \mathrm{m}$, diretamente nos frascos confeccionados em PTFE. Em seguida, foi adicionada uma mistura ácida contendo 9,0 $\mathrm{mL}$ de $\mathrm{HNO}_{3}$ e 3,0 mL de HF. Após, os frascos foram completamente fechados e encaminhados a um bloco digestor, no qual permaneceram por 3 horas a uma temperatura de $120^{\circ} \mathrm{C}$. Após o resfriamento do sistema, as soluções obtidas foram transferidas para frascos volumétricos de PP e o volume de 40,0 mL foi completado com uma solução de $\mathrm{H}_{3} \mathrm{BO}_{3} 4,5 \% \mathrm{~m} / \mathrm{v}$.

\section{RESULTADOS E DISCUSSÃO}

\section{Avaliação de massa e granulometria de amostra}

Para este estudo, uma amostra de louça cerâmica foi selecionada de forma aleatória e preparada seguindo o Procedimento 1. Três massas foram usadas para o preparo das amostras $(25,100$ e 250 $\mathrm{mg})$ em três granulometrias $(<25$, entre 25 e 53 e $53-150 \mu \mathrm{m})$ e o volume final das soluções foi de $20 \mathrm{~mL}$. Para este estudo, o volume de ácido foi fixado a fim de avaliar a quantidade máxima de amostra que poderia ser utilizada com um mesmo volume de ácido para o preparo.

A Figura 1 apresenta os resultados que foram obtidos para este estudo levando em consideração a concentração de amostra no final (massa/volume). O sinal de absorvância para cada analito foi normalizado em função da massa da amostra introduzida no atomizador $\left(\mathrm{Abs} \mathrm{g}^{-1}\right)$.

A condição que apresentou uma melhor sensibilidade, dada pelos maiores valores de absorvância obtidos, foi aquela em que se

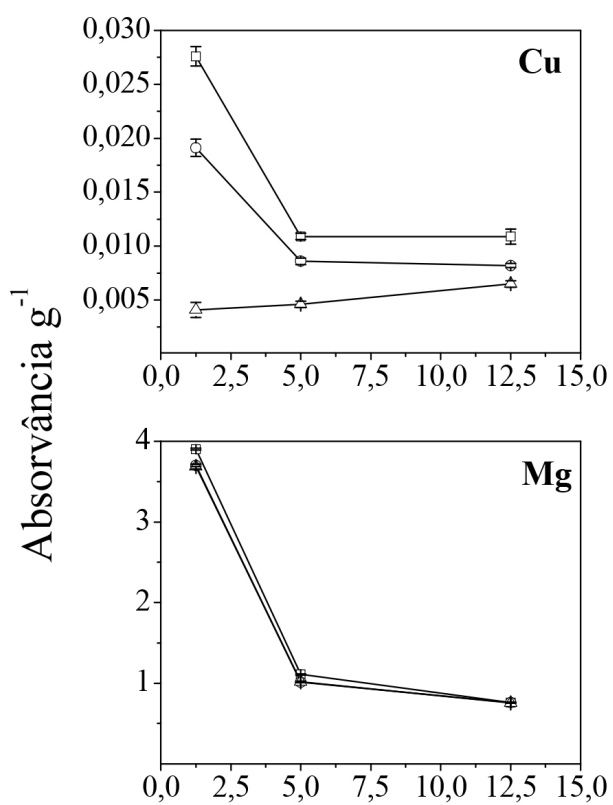

empregou a menor massa de amostra $(25 \mathrm{mg})$ e granulometrias menores que $53 \mu \mathrm{m}$. Já para as soluções com maiores massas (100 e 250 mg), foi obtida uma menor relação do sinal de absorvância por grama de amostra, principalmente para os analitos $\mathrm{Cu}, \mathrm{Mg}$ e $\mathrm{Zn}$. Esse fato pode ser explicado pelo aumento da presença da matriz da amostra, que pode causar um efeito no transporte da solução da amostra e, também, pode causar interferência de atomização devido ao aumento da quantidade de matriz no atomizador. De acordo com os resultados obtidos, as soluções foram preparadas com amostras cujas partículas eram inferiores a $25 \mu \mathrm{m}$, uma vez que, assim, é possível aumentar a sensibilidade, além de facilitar o processo de decomposição das amostras, melhorando a atomização dos analitos.

\section{Avaliação da concentração de ácido para o preparo das amostras}

Para o método de decomposição ácida assistida por ultrassom, fez-se a variação do volume de ácido para $25 \mathrm{mg}$ e, desse modo, da concentração de cada ácido empregado nas soluções finais, de acordo com as proporções apresentadas pela Tabela 2 .

Tabela 2. Proporções de $\mathrm{HNO}_{3}$ e de $\mathrm{HF}$ empregadas para a decomposição das amostras

\begin{tabular}{lcccc}
\hline Ensaio & $\mathrm{HNO}_{3}(\mathrm{~mL})$ & $\mathrm{HNO}_{3}(\%, \mathrm{~m} / \mathrm{v})$ & $\mathrm{HF}(\mathrm{mL})$ & $\mathrm{HF}(\%, \mathrm{~m} / \mathrm{v})$ \\
\hline 1 & & & 0,5 & 1,0 \\
2 & 0,5 & 1,6 & 1,0 & 2,0 \\
3 & & & 2,0 & 4,0 \\
\hline 4 & \multirow{2}{*}{1,0} & 3,2 & 0,5 & 1,0 \\
5 & & & 1,0 & 2,0 \\
6 & & & 2,0 & 4,0 \\
\hline 7 & \multirow{2}{*}{6,5} & 0,5 & 1,0 \\
8 & 2,0 & & 1,0 & 2,0 \\
9 & & & 2,0 & 4,0 \\
\hline
\end{tabular}
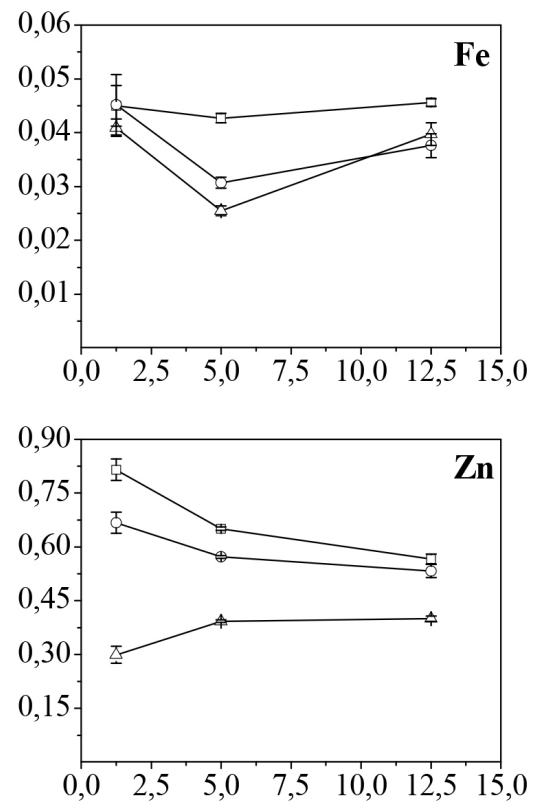

Concentração de Amostra $\left(\mathrm{g} \mathrm{L}^{-1}\right)$

Figura 1. Efeito da concentração de amostra no sinal de absorvância dos analitos $\mathrm{Cu}$, Fe, $\mathrm{Mg}$ e Zn em soluções de diferentes granulometrias: ( $\square$ ) <25 $\mu$; (O) entre 25 a $53 \mu \mathrm{me}(\triangle)$ entre 53 a $150 \mu \mathrm{m}$ 
A Figura 2 apresenta os resultados obtidos deste estudo para definir a melhor condição da mistura ácida a ser empregada para decomposições de amostras de materiais cerâmicos.
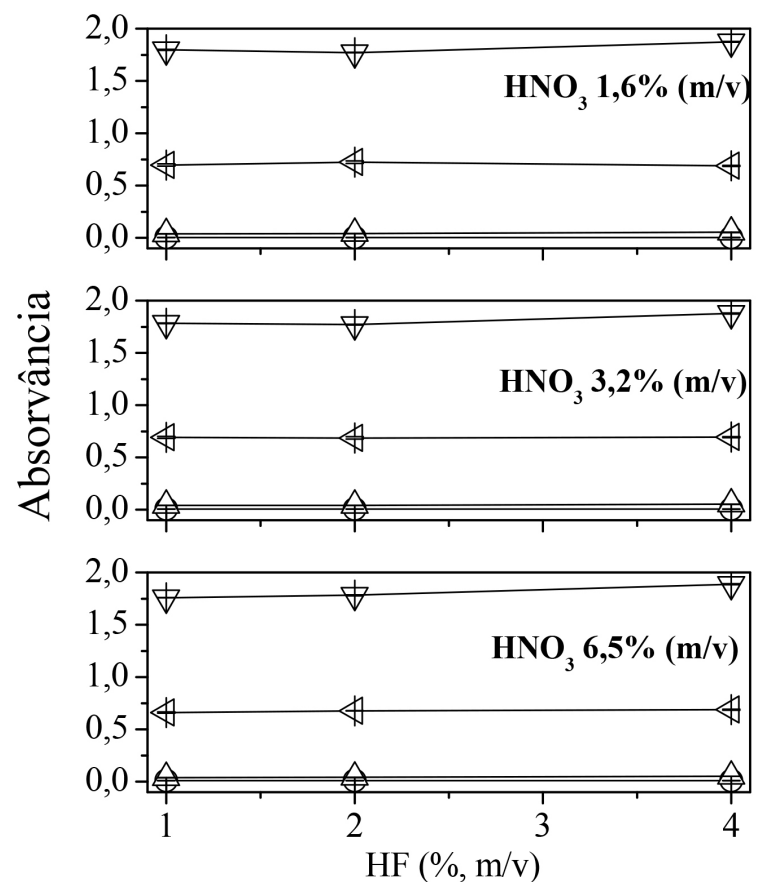

Figura 2. Efeito da concentração de $\mathrm{HF}$ e de $\mathrm{HNO}_{3}$ para o preparo de amostras de cerâmicas na determinação de $\mathrm{Cu}(\bigcirc), \mathrm{Fe}(\triangle), \mathrm{Mg}(\nabla)$ e $\mathrm{Zn}(\triangleleft)$

Verificou-se que não houve diferenças significativas no sinal de absorvância na medida em que a composição ácida foi variada para a determinação de $\mathrm{Cu}, \mathrm{Fe}, \mathrm{Mg}$ e $\mathrm{Zn}$. Dessa forma, para o preparo das amostras, foi adotada a menor concentração dos ácidos: 1,6\% $\mathrm{m} / \mathrm{v} \mathrm{HNO}_{3}$ e $1,0 \% \mathrm{~m} / \mathrm{v}$ HF. É importante ressaltar que sem o uso do HF não há uma completa decomposição, dado que as amostras de cerâmicas são ricas em silício, o que resultaria em um procedimento de extração.

Paralelamente, foi preparado um experimento no qual uma replicata da amostra foi deixada durante a noite à temperatura ambiente e na presença dos ácidos. Após pelo menos $12 \mathrm{~h}$ não foi observada nenhuma alteração, tendo em vista que permaneceu uma quantidade de sólido no fundo do frasco, comprovando, assim, a ação do ultrassom para a completa decomposição das amostras de cerâmicas.

\section{Avaliação da concentração de $\mathrm{CsCl} / \mathrm{LaCl}_{3}$ para a determinação de $\mathrm{Mg}$}

A ionização e a formação de compostos refratários são interferências do tipo não espectral e que podem ser consideradas comuns em análises por FAAS. Na interferência de ionização, os metais alcalinos e alcalinos terrosos, como o $\mathrm{Na}, \mathrm{K}, \mathrm{Ca}$ e $\mathrm{Mg}$, são considerados os elementos mais suscetíveis a interferências desta natureza, devido a seus baixos potenciais de ionização em relação aos demais elementos. Para a minimização dessa interferência, é possível adicionar junto às amostras e às soluções de calibração outro elemento mais facilmente ionizável (como, por exemplo, o Cs). Para as interferências de formação de compostos refratários, é comum a utilização de outro elemento capaz de formar, com o interferente, um composto mais estável. O elemento mais utilizado para esses casos é o La, conhecido como "agente liberador" justamente pelo fato de competir pelos compostos interferentes, liberando o analito de interesse para ser atomizado. ${ }^{11}$ Para a determinação de $\mathrm{Mg}$ em cerâmica foi avaliada a concentração a ser usada de $\mathrm{CsCl}$ e $\mathrm{LaCl}_{3}$ (solução de Schinkel) em soluções ácidas após a decomposição assistida por ultrassom, com diferentes concentrações de HF. Os resultados obtidos deste estudo estão apresentados na Figura 3.

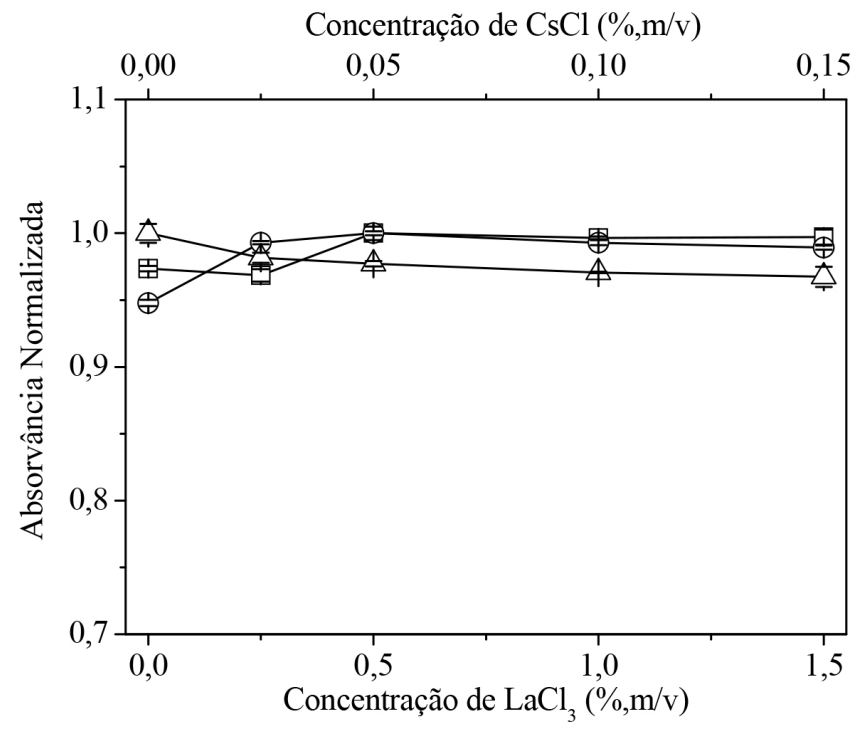

Figura 3. Absorvância de $\mathrm{Mg}$ em função da concentração de CsCl e $\mathrm{LaCl}_{3}$ em soluções de amostras de cerâmica em meio de ácido. Concentração de

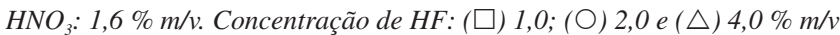

É possível perceber uma concordância entre as intensidades dos sinais para as diferentes soluções ácidas a partir da concentração de $0,05 \% \mathrm{~m} / \mathrm{v}$ de $\mathrm{CsCl}$ e $0,5 \% \mathrm{~m} / \mathrm{v}$ de $\mathrm{LaCl}_{3}$, mesmo variando a concentração de HF. Sendo assim, essa concentração foi escolhida para as posteriores determinações desse analito por F AAS.

\section{Concentração de $\mathrm{Cu}, \mathrm{Fe}, \mathrm{Mg}$, e $\mathrm{Zn}$ em cerâmicas de uso doméstico}

Após estabelecer as melhores condições para o preparo de decomposição ácida assistida por ultrassom (US), o método foi aplicado em oito diferentes amostras de louças cerâmicas para a determinação de $\mathrm{Cu}, \mathrm{Fe}, \mathrm{Mg}$ e $\mathrm{Zn}$ por $\mathrm{F}$ AAS. As amostras também foram preparadas usando os procedimentos 2 e 3, envolvendo a decomposição em sistema aberto (SA) e a decomposição em sistema fechado (SF), respectivamente. Os parâmetros de mérito obtidos para essas determinações estão dispostos na Tabela 3. A faixa linear de trabalho para os analitos foram: 1,0 a 4,0 $\mathrm{mg} \mathrm{L}^{-1}$ para $\mathrm{Cu} ; 1,0$ a $6,0 \mathrm{mg} \mathrm{L}^{-1}$ para Fe; 0,1 a $0,4 \mathrm{mg} \mathrm{L}^{-1}$ para $\mathrm{Mg}$ e 0,5 a $2,0 \mathrm{mg} \mathrm{L}^{-1}$ pra $\mathrm{Zn}$. Os valores de coeficiente de correlação linear foram todos maiores que 0,99 .

Verificou-se um menor limite de detecção (LD) e limite de quantificação (LQ) para a decomposição em sistema fechado, uma vez que, nesse procedimento, utiliza-se uma massa de amostra maior para a etapa de decomposição. Já para o método de decomposição ácida assistida por ultrassom, é requerida uma pequena quantidade de massa de amostra, o que reflete em limites de detecção e quantificação maiores, mas, ainda assim, extremamente adequados para as determinações dos analitos $\mathrm{Cu}, \mathrm{Fe}, \mathrm{Mg}$ e $\mathrm{Zn}$ nessas amostras.

Os resultados encontrados, em relação à concentração dos analitos nas amostras que foram preparadas pelos três métodos, estão apresentados na Tabela 4.

Para a determinação de $\mathrm{Cu}$ na amostra $\mathrm{A}$ e de $\mathrm{Zn}$ na amostra $\mathrm{C}$, ambas preparadas pelo método US, os valores obtidos ficaram abaixo do limite de detecção do analito quando se utilizou uma massa de 25 
Tabela 3. Parâmetros de mérito para a determinação de $\mathrm{Cu}, \mathrm{Fe}, \mathrm{Mg}$ e Zn por F AAS em amostras de cerâmicas de uso doméstico

\begin{tabular}{|c|c|c|c|c|c|c|c|c|}
\hline \multirow{2}{*}{ Analito } & \multirow{2}{*}{$\begin{array}{c}a \\
\left(\mathrm{~L} \mathrm{mg}^{-1}\right)\end{array}$} & \multirow[t]{2}{*}{$\mathrm{R}^{2}$} & $\begin{array}{c}\mathrm{LD} \\
\left(\mathrm{mg} \mathrm{g}^{-1}\right)\end{array}$ & $\begin{array}{c}\text { LQ } \\
\left(\mathrm{mg} \mathrm{g}^{-1}\right)\end{array}$ & $\begin{array}{c}\mathrm{LD} \\
\left(\mathrm{mg} \mathrm{g}^{-1}\right)\end{array}$ & $\begin{array}{c}\text { LQ } \\
\left(\mathrm{mg} \mathrm{g}^{-1}\right)\end{array}$ & $\begin{array}{c}\mathrm{LD} \\
\left(\mathrm{mg} \mathrm{g}^{-1}\right)\end{array}$ & $\begin{array}{c}\text { LQ } \\
\left(\mathrm{mg} \mathrm{g}^{-1}\right)\end{array}$ \\
\hline & & & \multicolumn{2}{|c|}{ Método US } & \multicolumn{2}{|c|}{ Método SA } & \multicolumn{2}{|c|}{ Método SF } \\
\hline $\mathrm{Cu}$ & 0,1299 & 0,9994 & 0,006 & 0,019 & 0,002 & 0,007 & 0,001 & 0,004 \\
\hline $\mathrm{Fe}$ & 0,0533 & 0,9977 & 0,019 & 0,063 & 0,007 & 0,024 & 0,004 & 0,013 \\
\hline $\mathrm{Mg}$ & 0,9071 & 0,9994 & 0,001 & 0,005 & 0,0005 & 0,002 & 0,0003 & 0,0009 \\
\hline $\mathrm{Zn}$ & 0,4969 & 0,9941 & 0,003 & 0,008 & 0,001 & 0,003 & 0,0005 & 0,002 \\
\hline
\end{tabular}

$a$ : Sensibilidade; $\mathrm{R}^{2}$ : Coeficiente de correlação linear; LD: Limite de detecção; LQ: Limite de quantificação. Método US: Decomposição assistida por ultrassom. Método SA: Decomposição em sistema aberto. Método SF: Decomposição em sistema fechado.

Tabela 4. Resultados em concentração de $\mathrm{Cu}, \mathrm{Fe}, \mathrm{Mg}$ e $\mathrm{Zn}$ encontrados em amostras de cerâmica, (n=3)

\begin{tabular}{|c|c|c|c|c|c|c|c|c|c|c|c|c|}
\hline \multirow{3}{*}{ Amostra } & \multicolumn{12}{|c|}{ Concentração $\overline{\mathrm{x}} \pm \mathrm{sd}, \mathrm{mg} \mathrm{g}^{-1}(\mathrm{RSD}, \%)$} \\
\hline & \multicolumn{3}{|c|}{$\mathrm{Cu}$} & \multicolumn{3}{|c|}{$\mathrm{Fe}$} & \multicolumn{3}{|c|}{$\mathrm{Mg}$} & \multicolumn{3}{|c|}{$\mathrm{Zn}$} \\
\hline & US & SF & SA & US & SF & SA & US & SF & SA & US & SF & SA \\
\hline A & $\begin{array}{c}0,019 \pm 0,002 \\
(10,5)^{*}\end{array}$ & $\begin{array}{c}0,019 \pm 0,001 \\
(5,2)\end{array}$ & $\begin{array}{c}0,020 \pm 0,001 \\
(5,0)\end{array}$ & $\begin{array}{c}8,04 \pm 0,10 \\
(1,2)\end{array}$ & $\begin{array}{c}8,10 \pm 0,21 \\
(2,6)\end{array}$ & $\begin{array}{c}8,01 \pm 0,65 \\
(8,1)\end{array}$ & $\begin{array}{c}2,78 \pm 0,03 \\
\quad(1,1)\end{array}$ & $\begin{array}{c}2,81 \pm 0,16 \\
(5,7)\end{array}$ & $\begin{array}{l}2,75 \pm 0,10 \\
(3,6)\end{array}$ & $\begin{array}{l}, 512 \pm 0,010 \\
(2,0)\end{array}$ & $\begin{array}{c}0,523 \pm 0,004 \\
(0,8)\end{array}$ & $\begin{array}{c}0,521 \pm 0,035 \\
(6,7)\end{array}$ \\
\hline B & $\begin{array}{c}0,081 \pm 0,001 \\
(1,2)\end{array}$ & $\begin{array}{l}0,082 \pm 0,001 \\
\quad(1,2)\end{array}$ & $\begin{array}{c}0,082 \pm 0,002 \\
(2,4)\end{array}$ & $\begin{array}{c}2,00 \pm 0,05 \\
(2,5)\end{array}$ & $\begin{array}{c}2,04 \pm 0,02 \\
(1,0)\end{array}$ & $\begin{array}{l}1,95 \pm 0,02 \\
(1,0)\end{array}$ & $\begin{array}{c}157,33 \pm 9,08 \\
\quad(5,8)\end{array}$ & $\begin{array}{c}154,20 \pm 3,06 \\
(2,0)\end{array}$ & $\begin{array}{c}153,68 \pm 2,74 \\
(1,8)\end{array}$ & $\begin{array}{c}2,677 \pm 0,061 \\
\quad(2,3)\end{array}$ & $\begin{array}{c}2,645 \pm 0,072 \\
(2,7)\end{array}$ & $\begin{array}{c}2,697 \pm 0,031 \\
(1,1)\end{array}$ \\
\hline$C$ & $\begin{array}{c}0,110 \pm 0,002 \\
(1,8)\end{array}$ & $\begin{array}{c}0,107 \pm 0,003 \\
(2,8)\end{array}$ & $\begin{array}{c}0,110 \pm 0,001 \\
(0,9)\end{array}$ & $\begin{array}{c}3,75 \pm 0,10 \\
(2,7)\end{array}$ & $\begin{array}{c}3,79 \pm 0,27 \\
(7,1)\end{array}$ & $\begin{array}{c}3,73 \pm 0,02 \\
(0,5)\end{array}$ & $\begin{array}{c}6,85 \pm 0,55 \\
(8,0)\end{array}$ & $\begin{array}{c}6,82 \pm 0,40 \\
\quad(5,9)\end{array}$ & $\begin{array}{c}6,77 \pm 0,16 \\
(2,4)\end{array}$ & $\begin{array}{c}0,066 \pm 0,002 \\
(3,1)^{*}\end{array}$ & $\begin{array}{c}0,065 \pm 0,002 \\
(3,1)\end{array}$ & $\begin{array}{c}0,064 \pm 0,001 \\
(1,6)\end{array}$ \\
\hline D & $\begin{array}{c}0,213 \pm 0,009 \\
(4,2)\end{array}$ & $\begin{array}{c}0,204 \pm 0,002 \\
(1,0)\end{array}$ & $\begin{array}{c}0,209 \pm 0,011 \\
(5,3)\end{array}$ & $\begin{array}{c}8,21 \pm 0,61 \\
(7,4)\end{array}$ & $\begin{array}{c}8,15 \pm 0,06 \\
(0,7)\end{array}$ & $\begin{array}{c}8,17 \pm 0,35 \\
(4,3)\end{array}$ & $\begin{array}{c}32,66 \pm 0,24 \\
(0,7)\end{array}$ & $\begin{array}{c}32,85 \pm 0,11 \\
(0,3)\end{array}$ & $\begin{array}{c}32,47 \pm 0,50 \\
(1,5)\end{array}$ & $\begin{array}{c}0,535 \pm 0,008 \\
(1,5)\end{array}$ & $\begin{array}{c}0,530 \pm 0,017 \\
(3,2)\end{array}$ & $\begin{array}{c}0,512 \pm 0,018 \\
(3,5)\end{array}$ \\
\hline E & $\begin{array}{c}0,163 \pm 0,003 \\
(1,8)\end{array}$ & $\begin{array}{c}0,164 \pm 0,001 \\
(0,6)\end{array}$ & $\begin{array}{c}0,166 \pm 0,008 \\
(4,8)\end{array}$ & $\begin{array}{c}3,45 \pm 0,08 \\
(2,3)\end{array}$ & $\begin{array}{c}3,57 \pm 0,06 \\
(1,7)\end{array}$ & $\begin{array}{c}3,48 \pm 0,05 \\
(1,4)\end{array}$ & $\begin{array}{c}3,65 \pm 0,15 \\
(4,1)\end{array}$ & $\begin{array}{c}3,56 \pm 0,04 \\
(1,1)\end{array}$ & $\begin{array}{c}3,58 \pm 0,28 \\
(7,8)\end{array}$ & $\begin{array}{c}1,029 \pm 0,058 \\
(5,6)\end{array}$ & $\begin{array}{c}1,095 \pm 0,019 \\
(1,7)\end{array}$ & $\begin{array}{c}1,081 \pm 0,009 \\
(0,8)\end{array}$ \\
\hline $\mathrm{F}$ & $\begin{array}{c}0,142 \pm 0,004 \\
(2,8)\end{array}$ & $\begin{array}{c}0,145 \pm 0,002 \\
(1,4)\end{array}$ & $\begin{array}{c}0,147 \pm 0,006 \\
(4,1)\end{array}$ & $\begin{array}{c}3,97 \pm 0,03 \\
(0,8)\end{array}$ & $\begin{array}{c}4,02 \pm 0,02 \\
(0,5) \\
\end{array}$ & $\begin{array}{c}3,98 \pm 0,07 \\
(1,8)\end{array}$ & $\begin{array}{c}6,64 \pm 0,09 \\
(1,4)\end{array}$ & $\begin{array}{c}6,75 \pm 0,31 \\
(4,6)\end{array}$ & $\begin{array}{c}6,84 \pm 0,12 \\
(1,8)\end{array}$ & $\begin{array}{c}0,135 \pm 0,004 \\
(3,0)\end{array}$ & $\begin{array}{c}0,133 \pm 0,006 \\
(4,5)\end{array}$ & $\begin{array}{c}0,137 \pm 0,006 \\
(4,4)\end{array}$ \\
\hline G & $\begin{array}{c}0,180 \pm 0,002 \\
(1,1)\end{array}$ & $\begin{array}{c}0,185 \pm 0,008 \\
(4,3)\end{array}$ & $\begin{array}{c}0,178 \pm 0,002 \\
(1,1)\end{array}$ & $\begin{array}{c}9,79 \pm 0,13 \\
(1,3) \\
\end{array}$ & $\begin{array}{c}9,64 \pm 0,31 \\
(3,2) \\
\end{array}$ & $\begin{array}{c}9,78 \pm 0,12 \\
(1,2) \\
\end{array}$ & $\begin{array}{c}31,01 \pm 0,29 \\
(0,9)\end{array}$ & $\begin{array}{c}31,11 \pm 0,74 \\
(2,4)\end{array}$ & $\begin{array}{c}31,33 \pm 0,44 \\
(1,4)\end{array}$ & $\begin{array}{c}0,298 \pm 0,021 \\
(7,0)\end{array}$ & $\begin{array}{c}0,322 \pm 0,014 \\
(4,3)\end{array}$ & $\begin{array}{c}0,294 \pm 0,015 \\
(5,1)\end{array}$ \\
\hline $\mathrm{H}$ & $\begin{array}{c}0,195 \pm 0,008 \\
(4,1)\end{array}$ & $\begin{array}{c}0,197 \pm 0,003 \\
(1,5)\end{array}$ & $\begin{array}{c}0,196 \pm 0,004 \\
(2,0)\end{array}$ & $\begin{array}{c}7,63 \pm 0,08 \\
(1,0)\end{array}$ & $\begin{array}{c}7,75 \pm 0,15 \\
(1,9)\end{array}$ & $\begin{array}{c}7,85 \pm 0,21 \\
(2,7)\end{array}$ & $\begin{array}{c}3,67 \pm 0,06 \\
(1,6) \\
\end{array}$ & $\begin{array}{c}3,66 \pm 0,04 \\
(1,1)\end{array}$ & $\begin{array}{c}3,66 \pm 0,06 \\
(1,6) \\
\end{array}$ & $\begin{array}{c}2,514 \pm 0,029 \\
(1,2) \\
\end{array}$ & $\begin{array}{c}2,506 \pm 0,137 \\
(5,5)\end{array}$ & $\begin{array}{c}2,501 \pm 0,089 \\
(3,6) \\
\end{array}$ \\
\hline
\end{tabular}

*massa de amostra: 100 mg. US: Decomposição assistida por ultrassom. SA: Decomposição em sistema aberto. SF: Decomposição em sistema fechado.

$\mathrm{mg}$. Essas amostras foram preparadas novamente pelo mesmo método e foi possível quantificar esse analito, uma vez que foi utilizada uma maior massa (aproximadamente $100 \mathrm{mg}$ ). Os resultados obtidos, portanto, foram concordantes com aqueles dos demais métodos utilizados, que utilizam maior massa de amostra. Dessa forma, é possível mostrar que o método proposto envolvendo a decomposição assistida por ultrassom é robusto, ou seja, é susceptível a variações experimentais, como no caso da variação de massa, propiciando uma melhora de 4 vezes nos valores de LD e LQ.

Para a comparação de métodos, foi aplicado o teste estatístico $t$, pareado para um limite de confiança de $95 \%$, pelo qual foi possível verificar que não há diferenças significativas entre os resultados encontrados com o método proposto envolvendo o uso do ultrassom em relação aos métodos comparativos (sistema aberto e sistema fechado). Com relação à precisão, todos os métodos apresentaram resultados próximos e com baixos valores de desvio padrão relativo (RSD, \%), uma vez que as médias desses desvios foram de 3,0; 2,7 e 3,0 para os métodos de US, SF e SA, respectivamente, confirmando que todos os procedimentos fornecem uma completa decomposição da matriz das amostras de cerâmica de uso doméstico.

Conforme os resultados apresentados na Tabela 4, de um modo geral, o $\mathrm{Cu}$ foi o elemento que apresentou as menores concentrações nas amostras de cerâmica de uso doméstico analisadas. Essa baixa concentração pode ser oriunda de algum pigmento utilizado para a decoração das louças.

Já o Mg foi obtido em uma concentração elevada em todas as amostras, quando comparado com os demais analitos determinados, mas com destaque para a amostra B, que apresentou uma concentração de aproximadamente $150 \mathrm{mg} \mathrm{g}^{-1}$ para esse analito. Essa concentração é de origem, possivelmente, da massa cerâmica industrial, que pode conter $\mathrm{Mg}$ em sua composição devido aos ingredientes que são adicionados durante o processo de produção (sais e óxidos) ou diretamente da matéria-prima (como, por exemplo, a magnesita). ${ }^{28-30}$ Além disso, a presença desse analito pode ser devido à adição de óxido de magnésio, que é vantajoso devido ao aumento da propriedade de refratariedade das peças. ${ }^{28-31}$

Para o $\mathrm{Fe}$, as concentrações encontradas mostraram-se próximas para todas as amostras, variando de 2,0 a 9,8 $\mathrm{mg} \mathrm{g}^{-1}$. Uma concentração baixa é esperada para esse analito, uma vez que o óxido de ferro pode influenciar na brancura do produto cerâmico queimado, afetando, assim, a eficácia dos pigmentos que são adicionados para dar coloração aos utensílios cerâmicos. ${ }^{32}$

Para Zn, duas amostras, B e H, apresentaram concentrações semelhantes e superiores às demais. Essa concentração pode estar associada ao esmalte cerâmico, que pode conter óxido de zinco como um de seus componentes. ${ }^{33}$

Todas as amostras avaliadas são fabricadas em indústrias distintas, o que explica a grande discrepância na concentração dos analitos quantificados. Isso se deve, ainda, a diferentes processos industriais utilizados por cada fabricante, incluindo desde a matéria prima, fundentes, ingredientes refratários, até as tintas utilizadas, o que acaba deixando os utensílios cerâmicos aparentemente iguais, contudo com composições químicas diferentes.

Para avaliar a exatidão do método proposto de decomposição 
Tabela 5. Concentrações de $\mathrm{Cu}, \mathrm{Fe}, \mathrm{Mg}$ e Zn em amostras de cerâmica, após a adição de diferentes concentrações

\begin{tabular}{|c|c|c|c|c|c|}
\hline \multirow{2}{*}{ Analito } & \multirow{2}{*}{ Adição, mg g ${ }^{-1}$} & \multicolumn{4}{|c|}{ Concentração $\overline{\mathrm{x}} \pm \mathrm{sd}, \mathrm{mg} \mathrm{g}^{-1}(\mathrm{RSD}, \%)$} \\
\hline & & Amostra A & Recuperação, \% & Amostra C & Recuperação, \% \\
\hline \multirow{3}{*}{$\mathrm{Cu}$} & 0,80 & $0,84 \pm 0,01(1,2)$ & 105 & $0,88 \pm 0,04(4,5)$ & 96,2 \\
\hline & 1,60 & $1,64 \pm 0,03(1,8)$ & 102,5 & $1,76 \pm 0,01(0,6)$ & 103,1 \\
\hline & 2,40 & $2,32 \pm 0,02(0,9)$ & 96,7 & $2,55 \pm 0,03(1,2)$ & 101,7 \\
\hline \multirow{3}{*}{$\mathrm{Fe}$} & 1,60 & $9,45 \pm 0,14(1,5)$ & 88,1 & $5,15 \pm 0,06(1,2)$ & 87,5 \\
\hline & 3,20 & $11,24 \pm 0,18(1,6)$ & 100,0 & $6,77 \pm 0,45(6,6)$ & 94,4 \\
\hline & 4,80 & $12,76 \pm 0,08(0,6)$ & 98,3 & $8,36 \pm 0,65(7,8)$ & 96,0 \\
\hline \multirow{3}{*}{$\mathrm{Mg}$} & 16,0 & $19,30 \pm 1,11(5,8)$ & 103,2 & $22,67 \pm 0,81(3,6)$ & 98,9 \\
\hline & 32,0 & $36,61 \pm 1,59(4,3)$ & 105,7 & $42,46 \pm 0,71(1,7)$ & 111,3 \\
\hline & 48,0 & $53,49 \pm 0,64(1,2)$ & 105,6 & $54,37 \pm 1,16(2,1)$ & 99,0 \\
\hline \multirow{3}{*}{$\mathrm{Zn}$} & 0,80 & $1,25 \pm 0,02(1,6)$ & 92,5 & $0,77 \pm 0,06(7,8)$ & 96,2 \\
\hline & 1,60 & $2,09 \pm 0,02(1,0)$ & 98,7 & $1,45 \pm 0,02(1,4)$ & 90,6 \\
\hline & 2,40 & $2,73 \pm 0,09(3,3)$ & 92,5 & $2,32 \pm 0,07(3,0)$ & 96,7 \\
\hline
\end{tabular}

ácida assistida por ultrassom, testes de adição e recuperação dos analitos foram realizados em três níveis de concentração. Ressaltamos que esse teste pode ser utilizado com segurança pelo método proposto, uma vez que foi assegurada a completa decomposição das amostras. Os resultados obtidos para a concentração total (amostra + adição) estão apresentados na Tabela 5.

Verificou-se que foram obtidas ótimas recuperações dos analitos adicionados, ficando em uma faixa de 87 a $112 \%$. Isso mostra que a exatidão do método usando a energia ultrassônica foi assegurada pelo procedimento acima, como também pelo estudo comparativo com outros procedimentos de preparo de amostras descritos na literatura e reconhecidos para a decomposição completa de amostras ricas em silicatos. Dessa forma, o método desenvolvido neste trabalho pode ser empregado para o controle de $\mathrm{Cu}, \mathrm{Fe}, \mathrm{Mg}$ e $\mathrm{Zn}$ em amostras de cerâmicas.

\section{CONCLUSÃO}

A metodologia apresentada mostrou-se com um grande potencial para a determinação de metais em amostras de cerâmica de uso doméstico. Embora o método necessite do emprego de ácidos concentrados, bem como que a amostra esteja finamente moída, viabiliza a utilização de condições mais brandas, possibilitando maior segurança, praticidade e uso de equipamentos de baixo custo. Além disso, mostrou resultados precisos e exatos, com base nos desvios padrões relativos obtidos, comparação entre os métodos, além do teste de adição e recuperação.

Com relação aos resultados das concentrações dos analitos nas amostras avaliadas, é possível ter indícios da composição química da matéria prima utilizada na fabricação de produtos cerâmicos, bem como de possíveis contaminações por elementos tóxicos. Sendo assim, essa metodologia se torna imprescindível de ser aplicada para o controle desse tipo de amostra, a fim de assegurar a qualidade do produto.

Os resultados obtidos apontam, certamente, que o método de preparo de amostra desenvolvido poderá ser aplicado nas determinações de outros elementos por técnicas analíticas mais sensíveis e com caráter multielementar, como ICP-MS e ICP OES, uma vez que ficou comprovado que, utilizando-se o ultrassom, ocorre a decomposição total e, assim, uma minimização de interferências durante as análises. Além disso, o método também poderá ser facilmente implementado em laboratórios de controle de qualidade e de fiscalização por parte do governo brasileiro para avaliar a qualidade dos produtos cerâmicos importados destinados ao uso doméstico.

\section{AGRADECIMENTOS}

Os autores agradecem à Agência Nacional de Vigilância Sanitária (ANVISA) e ao Conselho Nacional de Pesquisa Científica (CNPq), através da Chamada MCTI/CNPq/ANVISA No 23/2012 (Processo 403403/2012-0), pelo auxílio financeiro, e à Coordenação de Aperfeiçoamento de Pessoal de Nível Superior (CAPES) e ao CNPq pelas bolsas concedidas.

\section{REFERÊNCIAS}

1. Sômiya, S.; Aldinger, F.; Claussen, N.; Spriggs, R. M.; Uchino, K.; Koumoto, K.; Kaneno, M.; Handbook of advanced ceramics, vol. II: processing and theirs application, Elsevier: Amsterdam, 2003.

2. Omolaoye, J. A.; Uzairu, A.; Gimba, C. E.; Arch. Appl. Sci. Res. 2010, 2,120 .

3. Askeland, D. R.; Fulay, P. P.; Wright, W. J.; The science and engineering of materials, $6^{\mathrm{a}}$ ed., Cengage Learning: Boston, 2010.

4. Kolar, D.; Pure Appl. Chem. 2000, 72, 1425.

5. Montoya, C. P.; Historia de la cerâmica en el museo arqueológico nacional. Editora Ministerio de Cultura, Fundación Caja Madrid: Madrid, 1997, 32 p.

6. Navarro, R. F.; Revista Eletrônica de Materiais e Processos 2006, 1, 1.

7. Ruiz, M. S.; Tanno, L. C.; Cabral Junior, M.; Coelho, J. M.; Niedzielski, J. C.; Ceram. Ind. 2011, 16, 29.

8. Ministério de Minas e Energia (MME) - Relatório Técnico 74 - Perfil de louças sanitárias e de mesa, setembro de 2009.

9. Baracho, P. R.; Claudino, F. E.; Silva, C. S.; Casali, G. P.; Silva, V. L.; Longo, E.; Werber, I. T.; Ceram. Ind. 2012, 17, 39.

10. Camargo, A. C.; Portela, J. C. S.; Yoshimura, H. N.; Ceram. Ind. 2005, 10,39 .

11. Welz, B.; Sperling, M.; Atomic Absorption Spectrometry, $3^{\mathrm{a}}$ ed., WileyVCH: Germany, 1999.

12. Bing, N. H.; Bogaerts, A.; Broekaert, J. A. C.; Anal. Chem. 2010, 82, 4653.

13. Oliveira, E.; J. Braz. Chem. Soc. 2003, 14, 174.

14. Krug, F. J.; Métodos de preparo de amostras: Fundamentos sobre preparo de amostras orgânicas e inorgânicas para análise elementar, $1^{\mathrm{a}}$ ed., Piracicaba, 2010. 
15. Korn, M. G. A.; Boa Morte, E. S.; Santos, D. C. M. B.; Castro, J. T.; Barbosa, J. T. P; Teixeira, A. P.; Fernandes, A. P.; Welz, B.; Santos, W. P. C.; Santos, E. B. G. N.; Korn, M.; Appl. Spectrosc. Rev. 2008, 43, 67.

16. Callister, W. D.; Rethwisch, D. G. Materials Science and engineering: An introduction, $8^{\mathrm{a}}$ ed., John Wiley \& Sons: Versailles, 2009.

17. Xu, Y.; Iwashita, A.; Nakajima, T.; Yamashita, H.; Takanashi, H.; Ohki, A.; Talanta 2005, 66, 58.

18. Wang, J.; Topham, N.; Wu, C.; Talanta 2011, 85, 2655.

19. Marina, M. A.; López, M. C. B.; Anal. Chim. Acta 2001, 432, 157.

20. Tsolakidou, A; Garrigós, J. B.; Kilikoglou, V.; Anal. Chim. Acta 2002, 474, 177.

21. Rojas, F. S.; Ojeda, C. B.; Pavón, J. M. C.; Microchem. J. 2010, 94, 7.

22. Santos, E. J.; Herrmann, A. B.; Prado, S. K.; Fantin, E. B.; Santos, V. W.; Oliveira, A. V. M.; Curtius, A. J.; Microchem. J. 2013, 108, 233.

23. Bessler, K. E.; Souza, A. T.; Lemos, S. S.; Gomes, F. B.; Souza, E. J.; Quim. Nova 2010, 33, 1408.

24. Priego-Capote, F.; Castro, M. D. L.; J. Biochem. Biophys. Methods 2007, $70,299$.
25. Nascentes, C. C.; Korn, M.; Sousa, C. S.; Arruda, M. A. Z.; J. Braz. Chem. Soc. 2001, 12, 57.

26. Santos, H. N.; Capelo, J. L.; Talanta 2007, 73, 795.

27. United States Environmental Protection Agency (EPA). Method 3052: Microwave assisted acid digestion of siliceous and organically based matrices. 1996.

28. Shreve, R. N.; Brink Jr., J. A.; Indústrias de processos químicos, 4. ed., Guanabara Koogan: Rio de Janeiro, 1997, 717 p.

29. Motta, J. F. M.; Zanardo, A.; Cabral Jr., M.; Ceram. Ind. 2001, 6, 28.

30. Xavier, R. Y.; Santos, G. R.; Melchiades, F. G.; Boschi, A. O.; Ceram. Ind. 2008, 13, 29.

31. Amaral, L. F.; Salomão, R.; Frollini, E.; Pandolfelli, V. C.; Cerâmica 2007, 53, 368 .

32. Sanchez, E.; Orts, M. J.; Garcia-Ten, J.; Cantavella, V.; Ceram. Ind. 2001, 6, 15 .

33. Melchiades, F. G.; Lepri Neto, C.; Alves, H. J.; Boschi, A. O.; Ceram. Ind. 2009, 14, 23 